\title{
Incerteza em entropia ${ }^{1}$
}

\section{Uncertainty in entropy}

\author{
JOSÉ CARLOS MAGOSSI \\ Universidade Estadual de Campinas | UNICAMP \\ JOSÉ RENATO PAVIOTTI \\ Universidade Estadual de Campinas | UNICAMP
}

\begin{abstract}
RESUMO Claude E. Shannon, em seu artigo seminal de 1948, inicia a Teoria da Informação, precursora da era da informação, cujos impactos e ramificações se multiplicam, desde então, em ritmo acelerado. Entre os conceitos da Teoria Matemática da Comunicação de Shannon, o de entropia comporta a diferentes interpretações. Desse modo, objetiva-se neste texto expor algumas considerações históricas acerca do conceito de entropia, sem pretensões de completude, ao considerar a"incerteza" existente em sua definição. O trocadilho é proposital, pois estima-se que inovações científicas ou tecnológicas, em Teoria da Informação, se encontram no amplo escopo, e na descrição precisa, do conceito de entropia.
\end{abstract}

Palavras-chave comunicação - entropia - Shannon - informação.

\begin{abstract}
Claude E. Shannon, in his seminal paper of 1948, founded Information Theory, a theory that is the precursor of the information age, whose impacts and ramifications, since then, have steadily grown. Among the concepts of Shannon's Mathematical Theory of Communication, entropy is the one that holds the most diverging interpretations. Thus, our goal in this article is to develop some historical considerations on the concept of entropy, without any intention of exhausting the subject, working with the "uncertainty" in its definition. The pun is deliberate, since we argue that scientific or technological innovations in Information Theory stem from the interplay of the broad scope and precise descriptions of the concept of entropy.
\end{abstract}

Keywords communication - entropy - Shannon-information.

\section{Introdução}

Os trabalhos de R. J. E. Clausius (1822-1888) podem ser vistos como aqueles que deram origem ao ramo da Física denominado Termodinâmica² ${ }^{2}$ Nessa acepção, entropia lembra transformação de energia, ou, como se diz, quando da segunda lei da termodinâmica, perda de calor. 0 assunto é vasto e com inúmeras interações com o mundo real, haja vista esse termo ser utilizado em situações voltadas a processos de transformação de calor. Como há problemas interessantes relacionados à Termodinâmica e, também, como há ainda certa busca por solidificação dessa área, essa palavra acaba por ganhar tanto força como interrogações, quando associada a problemas egressos da Termodinâmica.

Se na Termodinâmica há elasticidade de interpretações, o que se pode dizer quando a palavra "entropia" é exportada para ser utilizada, de modo extensivo, na área geral de Comunicação. Desse modo, surge uma interação 
entre entropia sob a ótica da Termodinâmica e entropia sob a ótica da Informação ${ }^{3}$, tendo a estatística como "pano de fundo". Uma discussão sobre esse assunto é elaborada por Philippe Jacquet em seu texto Naissance de la Théorie de I'Information, em que faz uma concisa exposição sobre a Teoria da Informação e sobre a influência de Leon Brillouin na relação entre Informação, Termodinâmica e Ciência"

Mas qual o porquê da relação entre entropia e informação? Claude E. Shannon (1916-2001), que trabalhava nos laboratórios Bell (Bell Labs), publica, em 1948, um artigo sobre o que hoje se denomina Teoria da informaçã $0^{5}$. Em seu artigo de 1948, Shannon desenvolve um modelo matemático para comunicação, "A Mathematical Theory of Communication".. Inicialmente sua teoria parecia não ser muito compreendida, mas com o passar do tempo percebe-se sua "força" e ela torna-se aceita pela comunidade científica como precursora da era digital. Shannon, em seu trabalho, lança mão da palavra "entropia" para caracterizar, grosso modo, certa medida de informação associada à ideia de incerteza. Assim, o conceito de entropia começa a ganhar formas além daquelas advindas da Termodinâmica ${ }^{7}$. Isso pode ser constatado em "Fifty years of signal processing: The IEEE Signal Processing Society and its Technologies 1994-1998", de Frederik Nebeker ${ }^{8}$, em que se expõe 0 ano de 1948 como annus mirabillis ${ }^{9}$, em razão de sua importância na era da Informação. No entanto, como a existência humana se dá, num certo sentido, pelos processos de comunicação, é óbvio que inúmeras ramificações surjam desse modelo matemático, haja vista que, segundo Shannon-Weaver ${ }^{10}$, e também Umberto Eco ${ }^{11}$, a Teoria da Informação se vale de princípios comuns a outras disciplinas da ciência. Desse modo, áreas como Psicologia, Linguística, Física, Engenharia, Biologia etc. prontamente lançaram interpretações do modelo de Shannon adaptadas a cada área em particular ${ }^{12}$.

A plasticidade associada à ideia de comunicação, seja ou não via Teoria de Shannon, é útil para a ciência, pois auxilia na criação de contexto e interação entre os domínios do saber. No entanto, em algumas situações, um cuidado maior se deve ter para que não ocorram inconsistências relacionadas aos termos da teoria e, consequentemente, interpretações errôneas na busca por novos conceitos; ou ainda ambiguidades na tentativa de sedimentar os conceitos já estabelecidos. A proliferação de artigos voltados à Teoria da Informação cresceu em demasia após 1948, ao ponto de que algumas associações, e revistas científicas, terem decidido limitar o escopo de publicações ditas como da área de Teoria da Informação ${ }^{13}$. É importante notar que o excesso de áreas conexas com a Teoria da Informação vem também da profícua associação entre o conceito de entropia, a palavra "incerteza" e os processos de comunicação. Como consequência das investigações em Teoria da Informação, Termodinâmica, Estatística e Comunicação, e da asserção de que informação seja diretamente proporcional à entropia e que também informação e incerteza estejam extremamente ligadas ${ }^{14}$, facilmente se deduz que "algo" com alta entropia é "algo" muito incerto, com muita informação; e "algo" com baixa entropia é quase certo, pouca informação. Facilmente se percebem as inúmeras situações no dia a dia que podem valer-se dessa leitura ${ }^{15}$. Esse fato pode ser atestado no artigo "Information Theory and the Digital Age", ${ }^{16}$ em que se expõem as dificuldades passadas pelos editores de revistas da área de engenharia, no que se refere a publicações sobre Teoria da informação. Esse assunto tornou-se tão vasto que "qualquer coisa" se encontrava no escopo dessa teoria e, desse modo, passível de publicação. Os editores começaram então a determinar uma política que restringisse 0 tema dos artigos. É o que se passa hoje com, por exemplo a revista IEEE Information Theory. Vale citar:

Inúmeros artigos fracos baseados em áreas que "não são da engenharia" estavam sendo publicados na IRE Transactions daquela época. Pior ainda, pesquisadores deliberadamente introduziam as palavras "teoria da informação" e "Cibernética", como elas eram alternativamente denominadas, na esperança de atrair fundos. Essa cega tentativa de aplicar Teoria da Informação a cada coisa sob o sol criou muita controvérsia dentro do PGIT sobre quais deveriam ser os limites desse campo ${ }^{\underline{17}}$.

Essa polêmica levou os editores a discutir sobre qual seria o escopo das publicações em Teoria da Informação. Dois artigos foram importantes para, talvez, caracterizar o foco da revista tal como hoje em dia: 0 artigo de C. Shannon, "Bandwagon", 18 e 0 artigo de N. Wiener, "What is Information Theory", 19 os quais respondem os comentários elencados por De Rosa ${ }^{20}$, conforme citado em Aftab는. 
Nos dias atuais há muitas revistas que tratam desses assuntos, entre elas pode-se citar o Instituto de Engenheiros Eletricistas e Eletrônicos, IEEE — Institute of Electrical and Electronics Engineers, com a revista IEEE Information Theory Society ${ }^{22}$, que se dedica às publicações voltadas, grosso modo, à Teoria de Shannon. Há também a revista Entropy ${ }^{23}$, que aborda o conceito de entropia e suas inúmeras aplicações. Assim, tanto a Termodinâmica quanto a Teoria da Informação possuem ramificações que denotam uma plasticidade impressionante, se o foco é o conceito de entropia. Entende-se que esse é o ponto de intersecção que acaba por facilitar a diversificação de interpretações. Seja na Termodinâmica, seja na Teoria da Informação, esse conceito caracteriza essa vasta ramificação, seja ela científica ou tecnológica. Assim, o investimento em áreas tecnológicas voltadas à Teoria da Informação não deixa de ser um investimento que parte do conceito de entropia, guardadas, evidentemente, as peculiaridades de cada tópico específico que seja investigado. Por isso talvez não seja abusivo dizer que o conceito de entropia é um conceito antrópico ${ }^{24}$.

Ao levar em conta a complexidade do conceito de entropia na Termodinâmica e sua igual essência na Teoria da Informação, objetiva-se neste texto expor algumas considerações acerca desse conceito e expor aspectos históricos ligados ao seu surgimento. Neste caso, o objetivo é clarear as indagações existentes acerca da relação do conceito de entropia com informação. Acredita-se que isso possa auxiliar não somente na sofisticação desse conceito (de entropia), mas também na compreensão de problemas atuais cuja essência seja determinada por ele.

Entende-se que análises históricas com fins de esclarecimento de conceitos implicam diretamente processos de transformação científica, pois evitam a transmissão fragmentada de conceitos. Por exemplo, um dos pontos que comumente induzem a interrogações refere-se às razões que levaram Shannon a utilizar a palavra "entropia" em sua teoria. Essas análises abrem espaço para que outros caminhos possam ser investigados, e mais ainda, que seja possível, ao clarear a gênese do surgimento de um determinado conceito, elaborar inovações, sejam elas em tecnologia ou na ciência ${ }^{25}$.

Expõem-se também, neste artigo, considerações quanto à origem de alguns nomes, significados etc., voltados à Teoria da Informação. Outros pontos investigados são: a letra $H$ como símbolo de entropia, a primeira utilização do termo "Teoria da Informação", a primeira aparição do termo "Informação Mútua" e os inventores da Teoria da Informação. É evidente que todas essas considerações se associam diretamente ao conceito de entropia exibido por Shannon como "medida de informação"트.

Nas seções do artigo, expõe-se primeiramente uma seção sobre a origem da palavra "entropia" e sua utilização por Shannon. Segue-se com a discussão sobre a origem da letra $H$, bem como dos termos "Teoria da Informação" e "Informação Mútua". Discute-se também sobre os inventores da Teoria da Informação. Finaliza-se com uma seção sobre conclusões e outra com as notas e referências bibliográficas.

\section{Entropia}

Segundo 0. Toeplitz (1881-1940)끄, a busca pela gênese de um conceito serve não só para facilitar sua compreensão, mas também para fins pedagógicos, como estratégia de transmissão de informações, base do conhecimento científico. A busca pela trajetória histórica relativa ao conceito de entropia possibilita não somente seu entendimento, mas também sua inter-relação com outros domínios da ciência. Nesta seção inicia-se, sem pretensões de completude, uma exposição sobre o conceito de entropia, seguida de alguns comentários sobre a relação desse conceito com a ciência em geral. Após essa abordagem inicial entra-se no ponto principal desta seção, que é a discussão sobre entropia na Teoria da Informação e comentários acerca da atribuição, por Shannon, do nome "entropia" à medição de informação. 0 objetivo não é uma exposição completa e detalhista desse conceito, mas apenas um caminho que indique um contexto no qual estejam reunidas entropia e Teoria da Informação com fins de esclarecer problemas correlatos. 


\title{
Origem do nome "entropia"
}

A palavra "entropia" foi cunhada pela primeira vez por Rudolf Clausius (1822-1888) no trabalho "Abhandlungenüber die mechanische Wärmetheorie", de 1864-1865.

\begin{abstract}
Se procurarmos por um nome significativo para $S$, poderíamos dizer, semelhante à magnitude U, que éo conteúdo de calor e trabalho do corpo, cuja magnitude S é o conteúdo transformacional do corpo. Mas penso que seria melhor usar nomes para magnitudes que são importantes para a ciência nas línguas antigas, de modo que possam ser aplicados inalterados em todas as novas línguas; sugiro chamar magnitude $S$ de

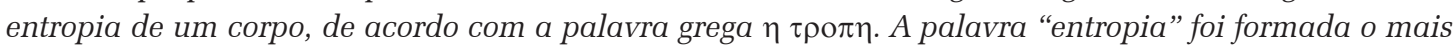
semelhantemente possível à palavra "energia", pois as duas magnitudes nomeadas por essas palavras estão tão relacionadas em seus significados físicos, que uma certa similaridade na designação me parece ser conveniente $e^{28}$.
\end{abstract}

Esse trabalho pode ser visto como a fundação da Termodinâmica. Nesse contexto, a palavra "entropia", do grego $\tau \rho о \pi \eta$, significa transformação. A primeira parte da palavra, en, lembra energia e a segunda parte lembra tropos, que significa retorno, mudança. Segundo Clausius,

$$
d S=\frac{d 0}{t}
$$

representa a entropia, como a variação do calor em relação ao tempo. A letra $S$ representa a entropia. Ludwig Boltzmann (1844-1906), que investigou o conceito de entropia na Termodinâmica, utiliza ainda a letra $S$ para entropia, mas fornece uma interpretação diferente. Para Boltzmann,

$$
S=k \log W \text {, }
$$

onde $W$ é o número de estados do sistema e $k$ é a constante de Boltzmannํㅗ․

\section{Entropia: um conceito complexo}

No Dicionário Aurélio, edição de 1999, a palavra "entropia" é referida como a "função termodinâmica de estado, associada à organização espacial e energética das partículas de um sistema, e cuja variação, numa transformação desse sistema, é medida pela integral do quociente da quantidade infinitesimal do calor trocado reversivelmente entre 0 sistema e o exterior pela temperatura absoluta do sistema" $\underline{\underline{30}}$. Nota-se que a ideia de entropia e teoria da informação não é contemplada. Por sua vez, o físico Alfred Wehrl, em seu artigo "General Properties of Entropy", 31 a situa na origem da Termodinâmica. A primeira lei da termodinâmica está presente nos trabalhos de Mayer (1842) e Joule (1845), e nos trabalhos de Clausius (1850) ${ }^{32}$ e Thomson (Lord Kelvin) (1852, 1857), os quais, fundamentados nos trabalhos de Carnot (1824), formularam o princípio da dissipação de energia e a segunda lei da termodinâmica. A noção de entropia foi, segundo Alfred Wehrl, introduzida por Clausius em 1865 ${ }^{33}$. Ainda no mesmo artigo, A. Wehrl discute a relação entre entropia na Termodinâmica e entropia na Teoria da Informação. Assunto esse também investigado por Leon Brillouin ${ }^{34}$. Mas a plasticidade desse conceito avança também em outras áreas. Em 1898, Marius Ameline faz um ensaio sobre a relação entre entropia e situações mentais no livro ${ }^{35}$ Énergie, entropie, pensée: Essai de psychophysique générale basée sur la thermodynamique avec un aperçu sur les variations de lientropie dans quelques situations mentales ${ }^{36}$ Nesse livro, em seus estudos, ele diz que: "As sensações aumentam sucessivamente a entropia dos neurônios, sua estabilidade ou sua inércia progressiva" ${ }^{\prime 37}$. Esse é um texto publicado em 1898, pouco tempo após Clausius ter escrito sobre a palavra "entropia". Nota-se, neste caso, a influência do conceito de entropia em campos diversos do conhecimento. Essa influência vai aumentar a partir da denominação de entropia, por Shannon, à medida de informação. 
Em síntese, o conceito de entropia surgiu na termodinâmica, com Clausius, para dizer de processos de transformação de calor, perda de calor em um sistema fechado; uma perda que não é mais recuperada, indicando um processo irreversível. Como Boltzmann utiliza, em sua formulação, a ideia de estados do sistema, a ideia de probabilidade, então, pode-se dizer desse conceito como denotando uma medida estatística ${ }^{38}$. Daí, seguindo os dizeres de Boltzmann, a ideia de irreversibilidade não se torna impossível, mas apenas improvável ${ }^{39}$. Como em Shannon, a ideia é a de medir informação aditiva contida em uma mensagem que denota as possíveis liberdades de escolha; então, a informação transmitida por uma mensagem pode ser representada pelo logaritmo binário do número de possibilidades que definem uma mensagem sem ambiguidades ${ }^{40}$.

Em Shannon-Weaver ${ }^{41}$, discute-se que o conceito de informação seja diretamente proporcional à entropia.

Nessa nova teoria, a palavra informação se refere não tanto a quanto se diz, mas a quanto se poderia dizer; isto é, a informação é a medida de nossa liberdade de escolha na seleção de uma mensagem [...]. Devemos recordar que na teoria matemática da comunicação nós não estamos interessados no significado das mensagens individuais, mas sim na natureza estatística global da fonte de informação [...]. O conceito de informação desenvolvido nessa teoria parece, a princípio, estranho e não satisfatório; insatisfatório porque não há nada a fazer com o significado e estranho porque não se refere a uma única mensagem, mas, ao contrário, ao caráter estatístico de um conjunto de mensagens; estranho, também, porque, em tais termos estatísticos, as palavras "informação" e "incerteza" estão extremamente ligadas ${ }^{\underline{42}}$.

Torna-se, então, evidente que as palavras "informação", "comunicação" e "estatística" impõem, de certa forma, um vasto leque de opções de leituras para o conceito de entropia. Talvez por isso o pesquisador Cédric Villani, em seu trabalho intitulado H-Theorem and beyond: Boltzmann's entropy in today's mathematics, diz do conceito de entropia como sendo uma noção antrópica, " [...] em particular o conceito de entropia implica uma observação, com alguma margem de erro. (Entropia é uma noção antrópica, [...])".

A literatura contempla uma vasta lista de artigos e livros relacionados aos conceitos de entropia e suas diversas ramificações. Não é objetivo neste artigo buscar completude nessa revisão bibliográfica. 0 livro Information Theory: 50 years of discovery ${ }^{44}$ contém inúmeras referências. Também é possível encontrar muitos textos e artigos em periódicos eletrônicos, tais como IEEE Xplore Digital Library, no endereço https://ieeexplore.ieee.org.

0 conceito de entropia hoje em dia é caracterizado por sua plasticidade única. Assim também o era na época de Shannon, momento em que ele escolheu o termo "entropia" para designar uma medida de informação. Para Shannon, a incerteza, ou entropia (letra $H$ ), de uma mensagem randômica $X$ sobre $n$ valores com probabilidade $p_{i}$, para $i=1,2$, $\ldots, n$ é definida como 45

$$
H(X)=-\sum_{i=1}^{n} p_{i} \log p_{i}
$$

Na Física, a entropia representa variação de calor, desordem de um sistema etc., e é representada pela letra $S$ na seguinte fórmula:

$$
S=k \log W
$$

Interessante é analisar as semelhanças entre as duas fórmulas, a de Boltzmann e a de Shannon:

$$
S=k \log W \quad H(X)=-\sum_{i=1}^{n} p_{i} \log p_{i}
$$


Para Shannon, entropia é uma medida de informação. No entanto, ao seguir os comentários sobre entropia, sob a ótica da física, torna-se sutil, complexo, associar entropia (no sentido da Física) com medida de informação (no sentido da Teoria da Informação). Com esse viés, facilmente se questiona o porquê da escolha, por Shannon, do nome "entropia" para medida de informação. Essa discussão será exposta na seção seguinte. Alguns autores escolhem outros nomes para designar a medida de informação de Shannon, tal como, por exemplo, o termo SMI, citado por Arieh Ben-Naim para representar Shannon's measure of information, ou seja, medida da informação de Shannon ${ }^{46}$.

\section{A escolha do nome "entropia" por Shannon}

Nas seções anteriores é possível perceber a inserção do conceito de entropia na ciência em geral e seus múltiplos comentários. Sem levar em conta a cronologia histórica anterior à data da promulgação, por Shannon, do nome "entropia" para unidade de informação, expõem-se frases de dois artigos que indicam confusões ${ }^{47}$ acerca da escolha desse nome por Shannon ${ }^{48}$. Há na literatura alguns questionamentos que parecem ser contraditórios. No artigo "Energy and Information", de M. Tribus e. C. Mclrvine de 1971는, os autores dizem que, em entrevista pessoal com Shannon, o próprio Shannon diz que o nome "entropia" advém de uma sugestão de Von Neumann, em conversa pessoal, e que ele, Shannon, prontamente aceitou.

O que há num nome? No caso da medida de Shannon, o nome não foi acidental. Em 1961 um de nós (Tribus) perguntou a Shannon o que ele tinha pensado quando finalmente confirmou sua famosa medida. Shannon respondeu: "Minha maior preocupação foi como chamá-la. Eu pensei em 'informação', mas a palavra foi excessivamente utilizada; assim, eu decidi chamá-la de 'incerteza'. Quando eu discuti isso com John von Neumann, ele teve uma ideia melhor. Von Neumann disse-me: 'Você deveria chamá-la de entropia, por duas razões. Em primeiro lugar, sua função de incerteza tem sido utilizada na mecânica estatística sob esse nome; assim, ela já tem um nome. Em segundo lugar, e mais importante, ninguém sabe o que realmente é entropia; assim, num debate você sempre tem vantagem, 50 ".

Já no artigo escrito por F. W. Ellersick, "A conversation with Claude Shannon", publicado em 198451, Shannon, dessa vez, mais velho, diz não saber dessa conversa.

R.P.: Tem sido dito que [John] Von Neumann sugeriu para você a palavra "entropia," dizendo para utilizá-la porque você vencerá toda vez porque ninguém a compreende e, além disso, ela prova p.log(p) perfeitamente ${ }^{52}$. Eu também escutei uma versão diferente dessa estória: que você deve ter chegado independentemente à palavra "entropia" e estava pensando em usá-la, mas era um tanto duvidoso, e você pediu conselhos para pessoas como Von Neumann e pessoas do laboratório Bell sobre se "entropia" poderia ser utilizada. Você já havia feito a identificação e, além disso, em seu relatório sobre criptografia de 1945, você utilizou a palavra "entropia"; você compara-a com mecânica estatística. Por outro lado, eu não acredito que você teve contato com Von Neumann em 1945. Assim, não me parece que Von Neumann tenha sugerido a palavra "entropia" para você.

C.S.: Não, eu não acho que ele sugeriu. Eu tenho certeza de que isso não aconteceu entre Von Neumann e e eu $u^{\underline{53}}$.

Desse modo, fica uma interrogação: Shannon seguiu os conselhos de John von Neumann ou não? 0 assunto é passível de investigação , no entanto, limita-se, neste texto, à simples exposição desses trechos de textos já publicados em revistas científicas . É evidente que, dada a complexidade desse conceito, essa escolha possa ter sido acertada por Shannon. Talvez sua genialidade tenha colocado uma incerteza na entropia (que não deixa de ser um conceito com viés de incerteza). Ou seja, há uma incerteza (entropia) também na escolha do nome "entropia". Esse trocadilho com a palavra incerteza é proposital, para indicar que ainda há incerteza, não somente na escolha de Shannon desse nome, mas uma "boa" incerteza em termos conceituais, haja vista que entropia é um conceito ainda em pleno desenvolvimento. 


\title{
As origens da letra $H$, Teoria da Informação e Informação Mútua
}

É comum questionar sobre a origem da letra $H$ utilizada por Shannon para representar a fórmula que calcula a entropia de uma mensagem. Alguns autores dizem que é a letra grega maiúscula eta, outros dizem que vem do teorema $H$ de Boltzmann. Com relação à letra $H$, em seu artigo de $1948^{\underline{54}}$ Shannon escreve: "A forma de $H$ será reconhecida como aquela da entropia tal como definida em certas formulações da mecânica estatística ${ }^{[8]}$ onde $p_{i}$ é a probabilidade de um sistema estar na célula $i$ de seu estado de fase. $H$ é então, por exemplo, o $H$ do famoso teorema $H$ de Boltzmann" $\underline{5}$.

A nota de rodapé número 8 dessa citação é: "See, for example, R. C. Tolman, Principles of Statistical Mechanics, Oxford, Clarendon, 1938". No entanto, ele não explicita o porquê da letra $H$, apenas indica que a tomou emprestada do famoso teorema de Boltzmann. Por outro lado, S. G. Brush, em seu livro Kinetic Theory ${ }^{\underline{56}}$, em nota de rodapé, escreve que:

Boltzmann ele mesmo originalmente utilizou a letra E, e não mudou para $H$ até 1895; o primeiro uso de H para essa quantidade foi aparentemente com S. H. Burbury, Phil. Mag. 30, 301 (1890). Um rumor tem circulado entre físicos modernos de que H era para ser a letra maiúscula eta, a letra eta que tinha sido usada para entropia por Gibbs e outros escritores, [ver Chapman, Nature 139, 931 (1937) ${ }^{57}$ ]. Isso dificilmente pode ser verdade, se de fato o uso foi originado por Burbury, já que ele não faz tal sugestão em seu artigo ${ }^{\frac{58}{}}$.

Ou seja, seguindo Brush, a utilização da letra $H$ para entropia se dá com Henry William Burbury no artigo “On some problems in the kinetic theory of gases", publicado em 1890ํㅗ․ Também há a mesma referência sobre a letra $H$, desta vez com comentários, em A. Wehrl:

\begin{abstract}
Algumas vezes, principalmente na literatura matemática, alguém utiliza a letra $H$ em vez de $S$ para entropia. Afirma-se que o $H$ deveria ser a letra maiúscula "eta"; no entanto, isso não é tão certo. Em todo caso, a letra $H$ foi introduzida por Burbury somente em 1890, enquanto Boltzmann originalmente utilizava "E". Em física, $H$ não é uma boa notação, em razão do risco de confusão com Hamiltoniano. O nome "entropia" é atribuído a Clausius (1865) e significa transformação ( escolhido para lembrar a palavra "energia", .60
\end{abstract}

A letra $H$, utilizada para entropia na Física, foi também utilizada por Shannon em seu trabalho principal, conhecido hoje em dia como Teoria da Informação. Esses nomes acabaram por ser estabelecidos na comunidade de cientistas. Interessante é que Shannon escreve, discretamente, apenas uma única vez o termo "Teoria da Informação" na página 11 do artigo "A Mathematical Theory of Communication": "Quantidades da forma $H=-\sum p_{i} \log p_{i}$ (a constante $K$ apenas equivale à escolha de uma unidade de medida) têm papel central na Teoria da Informação como medida de informação, escolha e incerteza" $\underline{61}$.

É possível inferir que, após essa inscrição em seu trabalho do nome "Teoria da Informação", Shannon tenha, de certa forma, batizado sua teoria. No entanto, o mesmo parece não acontecer com outro nome presente em sua teoria, o nome "Informação Mútua", que indica a quantidade de informação I(X;Y) que é transmitida através do canal.

$I(X ; Y)=H(X)+H(Y)-H(X, Y)$ pode ser calculada com base no conhecimento da entropia da fonte $H(X)$, da entropia do receptor $H(Y)$ e na média da incerteza do sistema de comunicação como uma todo $H(X, Y)$ b2. Sobre o nome "Informação Mútua" há alguns comentários interessantes. Em uma entrevista com Robert Fano, em 2013, Sergio Verdú pergunta sobre a origem do termo "Informação Mútua": "SV: Você criou o nome 'informação mútua'. RF: Sim. Não é normalmente conhecido que fui eu. Estou surpreso que você saiba disso" $\underline{\underline{6}}$. No entanto, no recente livro de Moser, página 141, é dito que a "informação mútua" foi utilizada pela primeira vez por Philip M. Woodward e lan L. Davies ${ }^{64}$ : 
O primeiro uso de I para informação foi de Philip M. Woodward e Ian L. Davies em [WD52]. [...] No entanto, eles não utilizaram o nome "informação mútua". O nome somente começa a aparecer entre 1954 e 1956. Em 1954, Mark Pinsker publicou um artigo em russo [Pin54] com o título "Informação mútua entre um par de processos randômicos gaussianos estacionários." [...] Shannon certamente utilizou o termo "informação mútua" no artigo sobre capacidade erro-zero em 1956 [Sha56] $]^{65}$.

Nota-se que, na citação acima, 0 artigo [WD52] corresponde ao artigo de Woodward e Davies ${ }^{66}$, 0 artigo [Pin54] refere-se ao artigo de Pinsker ${ }^{67}$ e 0 artigo [Sha56] é o artigo "The zero error capacity of a noisy channel", $\underline{68}$ de Shannon. Não se procura por completude nas descrições, mas sim exibir, didaticamente, aspectos históricos cotidianamente discutidos que sejam relevantes para um bom andamento de pesquisas, aulas etc. Há com certeza outras fontes e outras interrogações e confusões acerca de conceitos da Teoria da Informação. Entende-se que, graças ao acesso às informações, muito facilitado nos dias atuais, artigos, livros, textos não publicados (ou publicados e desconhecidos) acabam por vir à tona e, com isso, novas informações e testemunhos aparecem, favorecendo e esclarecendo aspectos importantes na história da ciência.

\section{"Inventores” da Teoria da Informação}

Ainda com relação à Teoria da Informação, surge a pergunta: "Foi Shannon seu inventor?" Na ciência, quando um conceito emerge, normalmente há outros pesquisadores pensando no mesmo conceito, ao mesmo tempo. Um caso clássico é o surgimento do Cálculo, descoberto por I. Newton e paralelamente por G. Leibniz. Assim, dizer de Teoria da Informação, ou Teoria de Shannon, é dizer das inovações propostas por Shannon em seu artigo de 1948. No livro de Shannon e Weaver ${ }^{69}$, há uma discussão sobre as origens do trabalho de Shannon. Nele, diz-se que o trabalho de Shannon, com relação à entropia como "informação ausente", tem sua origem nos trabalhos de Boltzmann de física estatística (1894). Cita-se também L. Szilard (Zeitschrift für Physik, vol. 53, 1925) como um trabalho em que se discute a ideia de informação em física, e também John von Neumann (Mathematische Grundlagen der Quantenmechanick [Fundamentos matemáticos da mecânica quântica], Berlim, 1932, cap. V). Mesmo no livro de Shannon e Weaver ${ }^{70}$, há uma descrição de que no trabalho de Shannon há a caracterização de uma medida para informação, entropia, teoremas de codificação de fonte, teoremas de codificação de canal, informação mútua e capacidade de canal. Desse modo, entende-se que esses conceitos devem estar presentes nos trabalhos, de forma bem clara, de todos os autores que partilhem com Shannon da invenção da Teoria da Informação. É evidente que alguns cientistas podem, mesmo indiretamente, ter lançado as ideias fundamentais da Teoria da Informação, mesmo que não tenham obtido um desfecho como aquele obtido por Shannon. Isso, segundo algumas interpretações, Ihes atribui também o ônus da invenção. Longe de entrar nesse patamar de investigação, longe de abrir espaço para um tópico, diga-se de passagem, interessante da história da ciência, neste texto enunciam-se apenas os nomes e as características daqueles que são citados como inventores da Teoria da Informação.

No artigo de Shannon픈 há uma indicação de Norbert Wiener e seu livro Cybernetics ${ }^{72}$, em seu trabalho. Vale citar a seguinte passagem:

Teoria da Comunicação é fortemente grata a Wiener por muito de sua teoria e filosofia básica. Seu clássico relatório NDRC, The Interpolation, Extrapolation and Smoothing of Stationary Time Series (Wiley, 1949), contém a primeira evidente formulação da teoria da comunicação como um problema estatístico, o estudo de operações sobre séries temporais. Esse trabalho, embora principalmente preocupado com predições lineares e problemas de filtragem, é uma importante referência colateral em conexão com o presente artigo. Nós podemos também fazer referência aqui ao Cybernetics de Wiener (Wiley, 1948), que lida com problemas gerais de comunicação e controle ${ }^{73}$. 
Ainda no artigo de Shannon, outra citação é feita referindo Wiener: “Crédito deve ser dado também ao professor N. Wiener, cuja elegante solução de problemas de filtragem e previsão de conjuntos estacionários tem consideravelmente influenciado o pensamento do escritor nesse campo"프. De modo análogo, Wiener também cita Shannon em seu livro Cybenetics ${ }^{75}$, e também no artigo "What is Information Theory?". 0 próprio Wiener escreve: "A Teoria da Informação tem sido identificada na opinião pública para denotar a teoria da informação por bits, tal como desenvolvida por Claude E. Shannon e por mim mesmo"끄.

Em muitos livros e artigos há os nomes de Shannon e Wiener como os inventores da Teoria da Informação. No entanto, é preciso cuidado com os dizeres. Por exemplo, Robert Ash escreve que a Teoria Estatística de Comunicação foi criada por Shannon e Wiener, mas com abordagens diferentes. Enquanto em Shannon há codificação de mensagens, em Wiener a codificação é ausente. Na sequência, para dizer do propósito de seu livro, R. Ash escreve: "Eu penso que é justo dizer que este livro se refere inteiramente à formulação de Shannon, isto é, o campo de conhecimento matemático que teve sua origem no artigo fundamental de Shannon de 1948. Isso é o que 'teoria da informação' significa para nós "푸

Vale citar também John R. Pierce:

Tanto Wiener como Shannon consideram não o problema de um simples sinal, mas o problema de tratamento adequado com quaisquer sinais com base em um grupo ou conjunto de sinais possíveis. Havia uma livre troca entre vários trabalhadores antes da publicação ou do livro de Wiener ou do artigo de Shannon, e ideias similares e expressões aparecem em ambos, embora a interpretação de Shannon pareça ser única. Essencialmente, o nome de Wiener acaba por estar associado com a área de extração de sinais de um conjunto com base em ruídos de um determinado tipo. [...] O nome de Shannon acaba por estar associado com questões de codificação de mensagens escolhidas com base em um conjunto conhecido e que elas podem ser transmitidas precisamente e rapidamente na presença de ruídos ${ }^{79}$.

Nota-se nos comentários acima que há uma predominância de que Shannon e Wiener foram os "inventores" da Teoria da Informação. No entanto, fica claro também que a abordagem de Shannon difere da abordagem de Wiener. Em Shannon há a preocupação da transmissão de mensagens com uma codificação apropriada. Essa codificação pode ser feita de tal forma que se torne possível aproximar-se da capacidade de canal. Mas um ponto importante, ainda no livro de Shannon e Weaver $\stackrel{80}{ }$, refere-se ao fato de os trabalhos de Shannon estarem relacionados com ideias desenvolvidas por pesquisadores (ao redor de 20 anos antes de Shannon) tais como: H. Nyquist e R.V. L. Hartley. Esses comentários podem ser encontrados também no livro ${ }^{81}$ que é uma tradução francesa do original de Shannon e Weaver.

Supõe-se que haja uma fonte que produz mensagens de um determinado tipo e cujo canal de comunicação contém ruídos. Como representar ou codificar essas mensagens de acordo com a fonte que as produz por meio de sinais elétricos de tal forma que se obtenha a transmissão mais rápida possível na presença de um canal com ruídos? Qual é a máxima velocidade de transmissão no canal sem erros? Esses são os problemas que Shannon propôs e resolveu. Por isso, na literatura, cita-se Wiener e Shannon, mas indica-se que a abordagem de Shannon se volta à obtenção de melhorias na transmissão de informações.

A fórmula de Shannon ${ }^{82}$ para capacidade de canal, , digamos, obra prima do trabalho de Shannon, coincide ter ocorrido, em outras formas, também em trabalhos de outros pesquisadores. No trabalho de Rioul e Magossi ${ }^{83}$ investigam-se as fórmulas similares à de Shannon que ocorreram no mesmo ano de 1948. Pesquisadores como Jacques Laplume (abril de 1948), Stanford Goldman (maio de 1948), Willian G. Tuller (junho de 1948), C. Earp (junho de 1948), André Clavier (dezembro de 1948), Norbert Wiener (em seu livro de 1948) e Herbert Sullivan (não publicada) ${ }^{84}$, também obtiveram desenvolvimentos semelhantes aos de Shannon. Por esse motivo, entre outros, entende-se que o ano de 1948 tenha se tornado o Annus Mirabilis, conforme observado por Frederik Nebeker em seu artigo "Fifty years of signal processing: The IEEE Signal Processing Society and its Technologies 1994-1998". informação; no entanto, entropia é classicamente utilizada em termodinâmica e em mecânica estatística, que são mais antigas que a Teoria da Informação. Como já foi mencionado, o físico L. Szilard em 1929 utilizou a ideia de informação 
para resolver problemas da Física. Por outro lado, a medida da quantidade de informação fornecida por dados sobre parâmetros desconhecidos de R. A. Fisher ${ }^{86}$ é a primeira utilização de "informação" em matemática estatística, introduzida especialmente para a teoria de estimação estatística ${ }^{87}$. Isso pode levar a deduzir-se que a Teoria da Informação, em termos de conceitos e intuição, tem suas raízes matemáticas nos conceitos de desordem (ou entropia) na termodinâmica e na mecânica estatística ${ }^{88}$. As palavras "entropia" e "informação" carregam em suas etimologias uma diversidade de interações com a ciência em geral. Com isso, é justificável que interpretações diversas possam ser inferidas.

Talvez tenha sido esse o motivo que levou C. Shannon e W. Weaver a caracterizar a Teoria Matemática da Comunicaçã $0^{\frac{89}{9}}$, quando trocam a letra "A", artigo indefinido em inglês, do artigo de Shannon, "A Mathematical Theory of Communication" por "The", artigo definido em inglês, no livro The Mathematical Theory of Communication. Nesse livro, o primeiro capítulo é dedicado a uma visão extensiva da Teoria Matemática da Comunicação e suas possíveis implicações práticas e tecnológicas. A partir do segundo capítulo encontra-se a Teoria de Shannon. Ainda sob o viés de alertar sob as possíveis incertezas em Teoria da Informação, mais um comentário se faz, nesse artigo, com relação a esse livro. Uma leitura descuidada pode levar o leitor a confundir a teoria de Shannon com o primeiro capítulo do referido livro. Essa confusão pode levá-lo a confusões acerca do que é a matemática presente na Teoria de Shannon. Por esse motivo é que em muitos textos e artigos se encontra a denominação dos inventores da Teoria da Informação como sendo Shannon-Weaver. 0 livro La Théorie Mathématique de la Communication, lançado pela editora Cassini, França $a^{90}$, tem, no prefácio do professor Olivier Rioul, a explicação dessa comum confusão. Nesse livro, toma-se 0 cuidado de deixar como último capítulo aquele que diz da plasticidade da Teoria da Informação (capítulo primeiro na edição de 194991).

\section{Conclusão}

0 objetivo neste artigo foi o de expor uma leitura acerca da extensão do conceito de entropia, e seus conceitos emergentes, bem como sua magnífica inserção, conforme Shannon, na Teoria da Informação. Objetivou-se, também, mostrar o quanto a clarificação dos conceitos na gênese do descobrimento é importante para que a ciência avance sem a proliferação excessiva de contradições. A ciência evolui, seja pelas inovações tecnológicas que indicam novos aparatos com fins de melhorar a qualidade de vida das pessoas, seja pelas publicações que formam um agregado, complexo, diga-se de passagem, de conceitos e teorias que são utilizados para a produção científica. 0 momento atual é rico em termos de informações, haja vista que seu acesso é facilitado. Inúmeras revistas online, livros em bibliotecas, artigos em plataformas virtuais etc. favorecem imensamente a pesquisa científica. Talvez seja um momento único na história da ciência. Isso possibilita que as informações corretas, os fatos reais, possam ser obtidos. Com base nisso é que se fez a exposição deste artigo, com o objetivo de clarear, de fornecer uma leitura apropriada, e particular, dos assuntos voltados à Teoria da Informação, os quais se conectam com o conceito de entropia. Longe de buscar completude na exposição, estima-se que portas possam ser abertas para que outras informações possam ser adquiridas, de modo a completar, ou mesmo ajustar, as informações aqui expostas. A leitura particular dos temas neste artigo teve como objetivo principal esclarecer alguns pontos, os quais se entende que possam ser confusos. Com isso, estima-se que as informações possam ser divulgadas com presteza. A incerteza da entropia, tal como exposto neste artigo, talvez possa revelar mais do que um simples trocadilho de palavras; talvez revele que o conceito de entropia seja um conceito criado pela ação humana e sem paralelo na natureza. 


\section{Notas e referências bibliográficas}

José Carlos Magossi é doutor em Engenharia Elétrica e professor da Faculdade de Tecnologia da Universidade Estadual de Campinas FT/UNICAMP, Limeira/SP. E-mail: magossi@ft.unicamp.br.

José Renato Paviotti é mestrando do programa de pós-graduação da Faculdade de Tecnologia da UNICAMP FT/UNICAMP, Limeira/SP e servidor técnico-administrativo do Instituto Federal de Educação, Ciência e Tecnologia de São Paulo (IFSP), que aproveita para agradecer o apoio recebido do IFSP por meio dos programas de autocapacitação e incentivo educacional. E-mail: jrpaviotti@gmail.com.

1 Uma versão preliminar desta pesquisa foi apresentada em pôster no X Workshop da pós-graduação da FT/Unicamp, e publicada online nos anais do evento: PAVIOTTI, José Renato; MAGOSSI, José Carlos. Considerações sobre o conceito de entropia na Teoria da Informação. In: WORKSHOP DA PÓSGRADUAÇÃO DA FACULDADE DE TECNOLOGIA, 10, 2018, Limeira/SP. Anais 2018, 2018, p. 24. Disponível em: < https://wordpress.ft.unicamp.br/ workshoppos/edicao-2018/>. Acesso em: 30 out. 2018

2 CLAUSIUS, Rudolf. Abhandlungen über die mechanische Wärmetheorie. Braunschweig, Druck Und Verlag Von Friedrich Vieweg Und Sohn. 1864. Disponível em: <https://gallica.bnf.fr/ark:/12148/bpt6k95149n/f47.image> . Acesso em: 28 out. 2018. BEN-NAIM, Ariel. Discovery Entropy and the Second Law of Thermodynamics. A Playful Way of Discovering a Law of Nature. World Scientific, London, 2010.

3 BRILLOUIN, Leon. Science and Information Theory. First Published in 1956 by Academic Press, Inc, New York: Dover Publications, 2013. JAYNES, Edwin T. Information Theory and Statistical Mechanics, II. Physical Reviews, v. 108, 171-190, 1956.

4 JACQUET, Philippe. Naissance de la théorie de l'information, Bibnum [online], Calcul et informatique, mis en ligne le 01 septembre 2009. Disponível em: $<$ http://journals.openedition.org/bibnum/568>. Acesso em 16 de julho de 2018.

5 É comum também dizer Teoria de Shannon. Além do monumental artigo de 1948, outro artigo publicado por Shannon, A symbolic analysis of relay and switching circuits, é considerado um dos trabalhos de mestrado mais importantes do século XX. Com ele surgiram as facilidades da relação entre circuitos digitais e a era dos computadores. SHANNON, Claude E. Symbolic Analysis of Relay and Switching Circuits. Transactions American Institute of Electrical Engineers, v. 57, 713-723, 1938. MOSER, Stefan M.; CHEN, Po-Ning. A Student's Guide to Coding and Information Theory. Cambridge University Press, 2012.

6 SHANNON, Claude E. A Mathematical Theory of Communication. Bell System Technical Journal, v. 27, pp. 379-423, 623-656, 1948.

7 BRILLOUIN, op. cit., 2013 ; JAYNES, op. cit., 1956.

8 NEBEKER, Frederik. Fifty years of signal processing: The IEEE Signal Processing Society and its Technologies 1994-1998. The IEEE Signal Processing Society, 1998. Disponível em: < https://signalprocessingsociety.org/uploads/history/history.pdf>. Acesso em: 29 out. 2018.

9 A expressão Annus Mirabilis (Ano Admirável) é atribuída na história da ciência aos anos nos quais foram realizadas importantes descobertas que afetaram profundamente a humanidade. Dentre eles, 0 ano de 1905, com Albert Einstein, e 1666, com Isaac Newton. 0 ano de 1948 pode ser considerado Annus Mirabilis graças ao trabalho de Shannon e de outros grandes inventores que contribuíram para o surgimento e consolidação da Teoria da Informação. Idem.

10 SHANNON, Claude E.; WEAVER, Warren W. The Mathematical Theory of Communication. University of Illinois Press, Urbana, IL, 1949.

11 ECO, Umberto. A Estrutura Ausente. Editora Perspectiva, São Paulo, 2007.

12 NEBEKER, op. cit., 1998. AFTAB, 0. et al. Information Theory and the Digital Age. The Structure of Engineering Revolutions, Massachusetts Institute of Technology, Cambridge, 2001. Disponível em: < http://web.mit.edu/6.933/www/Fall2001/Shannon2.pdf>. Acesso em: 29 out. 2018. COVER, Thomas M.; THOMAS, Joy A. Elements of Information Theory. John Wiley \& Sons, 2012. VERDÚ, Sergio. Fifty years of Shannon Theory. IEEE Transactions on Information Theory, v. 44, n. 6, p. 2057-2078, 1998

13 NEBEKER, op. cit., 1998

14 SHANNON; WEAVER, op. cit., 1949. ECO, Umberto. Obra Aberta. Editora Perspectiva, 10.ed., São Paulo, p.143-146, 2015

15 BRILLOUIN, 2013, op. cit. ECO, 2007, op. cit. ECO, 2015, op. cit. DION, Emmanuel. Invitation à la théorie de l'information. Éditions du Seuil. Série Sciences. Paris, 1997.

16 AFTAB, op. cit., 2001

17 AFTAB, op. cit., 2001, p. 9-11. [tradução nossa].

18 SHANNON, Claude E. The Bandwagon. IRE Transactions on Information Theory, v. 2, n. 1, p. 3, 1956.

19 WIENER, Norbert. What is Information Theory. IRE Transactions on Information Theory, v. 48, 1956.

20 DE ROSA, L. A. In Which Fields Do We Graze?. IRE Transactions on Information Theory, v. 1, n. 3, p. 2, 1955

21 AFTAB, op. cit., 2001

$22<$ http://www.itsoc.org $>$.

$23<$ http://www.mdpi.com/journal/entropy $>$.

24 VILLANI, Cédric. H-Theorem and beyond: Boltzmann's entropy in today's mathematics, 2008. Disponível em: < http://docplayer.net/30183507-H-theoremandbeyond-boltzmann-s-entropy-in-today-s-mathematics.html>. Acesso em: 29 out. 2018.

25 KLEIN, Felix. Elementary Mathematics from an Advanced Standpoint. Arithmetic, Algebra and Analysis. Dover Publications, New York, 2004. KLEIN, Felix. Elementary Mathematics from an Advanced Standpoint. Geometry. Dover Publications, New York, 2004. TOEPLITZ, Otto. The Calculus - A Genetic Approach. Chicago University Press, Chicago, 2007.

26 SHANNON, op. cit., 1948, p. 10.

27 TOEPLITZ, op. cit., 2007.

28 CLAUSIUS, 1864, op. cit., p. 34, tradução nossa. Disponível em: < https://archive.org/details/abhandlungenber00claugoog/page/n432> Acesso em: 18 nov. 2018. A tradução para a língua inglesa deste trecho está disponível em: CLAUSIUS, R.; HIRST, T. The Mechanical Theory of Heat: With Its Applications 
to the Steam-engine and to the Physical Properties of Bodies. J. Van Voorst, 1867. Disponível em: < https://books.google.com.br/books?id=8LIEAAAYA AJqprintsec $=$ frontcover\&hl $=$ pt- BR\&source $=$ gbs_ge summary $r \& c a d=0 \# v=$ onepage\&q=entropy\&f=false $>$. Acesso em: 20 nov. 2018. p. 357.

29

30

31

32

33

34

35

AMELINE, Marius. Énergie, entropie, pensée: Essai de psychophysique générale basée sur la thermodynamique avec un aperçu sur les variations de lientropie dans quelques situations mentales. G. Carré et C. Naud, 1898. Disponível em: < https://gallica.bnf.fr/ark:/12148/bpt6k63045190?rk=64378;0> . Acesso em: 30 out. 2018.

36 Idem, tradução nossa.

37 AMELINE, op. cit., 1898, p. 96. [Tradução nossa].

38 Uma das grandes descobertas de Ludwig Boltzmann foi a fórmula $S=k \log W(1877)$, que conecta a variável de estado — "entropia" — com a quantidade de caos (ou desordem) de um sistema, que significa o número de microestados, número esse denotado como "probabilidade termodinâmica", que em alemão se escreve thermodynamische Wahrscheinliehkeit, representado pela letra W. WEHRL, 1978, op. cit., p. 223.

39

ECO, op. cit., 2015, p. 133

Idem, pp. 128-146.

SHANNON; WEAVER, op. cit., 1949.

ECO, op. cit., 2015, p. 145 Apud SHANNON; WEAVER, op. cit., 1949.

VILLANI, op. cit., 2015, p. 4 [Tradução nossa]

VERDÚ, Sergio; MCLAUGHLIN, Steven W. Information theory: 50 years of discovery. IEEE Press, 2000.

SHANNON, op. cit., 1948.

BEN-NAIM, op. cit., 2010, p. 11

7 Arieh Ben-Naim faz um relato histórico, com referências, sobre essa questão em $<$ http://www.eoht.info/page/Neumann - Shannon + anecdote $>$. Acesso em: 3 set. 2019.

RIOUL, Olivier. Shannon et la théorie de l'information. Analyse de la publication de C. E. Shannon, une théorie mathématique de la communication, 2018. Disponível em:http://www.bibnum.education.fr/calcul-informatique/theorie-de-l-information/une-theorie-mathematique-de-la-communication $>$. Acesso em: 19 nov. 2018.

49 TRIBUS, Myron; MCIRVINE, Edward C. Energy and information. Scientific American, v. 225, n. 3, p. 179-190, 1971.

50 TRIBUS, op. cit., 1971, p. 180, tradução nossa.

51 ELLERSICK, Fred W. A conversation with Claude Shannon. IEEE Communication Magazine, v. 22, n. 5, pp. 123-126, 1984.

52 LEVINE, Raphael D; TRIBUS, Myron. Maximum entropy formalism. In: Maximum Entropy Formalism Conference (1978: Massachusetts Institute of Technology). MIT Press, 1979. CAMPBELL, Jeremy. Grammatical man: Information, entropy, language, and life. New York: Simon and Schuster, 1982.

53 ELLERSICK, 1984, op. cit., p. 124. [tradução nossa].

54 SHANNON, op. cit., 1948.

55 SHANNON, op. cit., 1948, p. 11. [Tradução nossa].

56 BRUSH, Stephen G. Kinetic Theory, v. 1. The Nature of Gases and Heat. Irreversible Processes. American Journal of Physics, v. 34, n. 12, p. 1.205, 1.966.

57 CHAPMAN, S. Boltzmann's H-Theorem. Nature, v. 139, n. 3526, p. 931, 1937. Disponível em: < http://www.nature.com/nature/journal/v139/n3526/ abs/139931a0.html\#top >. Acesso em: 18 nov. 2018.

58 BRUSH, op. cit., 1966, p. 6. [Tradução nossa].

59 BURBURY, Samuel Hawksley. XXXV. On some problems in the kinetic theory of gases. The London, Edinburgh, and Dublin Philosophical Magazine and Journal of Science, v. 30, n. 185, pp. 298-317, 1890. Disponível em: < http://www.tandfonline.com/doi/pdf/10.1080/14786449008620029\#preview> ou <http://www.nature.com/nature/journal/v139/n3526/abs/139931a0.html\#top >. Acesso em: 30 out. 2018. CHAPMAN, 1937, op. cit.

60 WEHRL, op. cit., 1978, p. 223. [Tradução nossa].

61 SHANNON, op. cit., 1948, p. 11. [Tradução nossa].

62 REZA, Fazlollah M. An introduction to information theory. New York: McGraw-Hill, 1961.

63 VERDÚ, Sergio. A conversation with Robert Fano. IEEE Information Theory Society Newsletter, 2013, p. 8, [Tradução nossa].

64 WOODWARD, Philip M.; DAVIES, lan L. Information theory and inverse probability in telecommunication. Proceedings of the IEE-Part III: Radio and Communication Engineering, v. 99, n. 58, p. 37-44, 1952.

65 MOSER; CHEN, op. cit., 2012, p. 140-141. [Tradução nossa].

66 WOODWARD; DAVIES, op. cit., 1952. 
PINSKER, Mark S. Mutual information between a pair of stationary Gaussian random processes. In: Dokl. Akad. Nauk. USSR, 1954, p. 213-216.

68 SHANNON, Claude. The zero error capacity of a noisy channel. IRE Transactions on Information Theory, v. 2, n. 3, p. 8-19, 1956.

69 SHANNON; WEAVER, op. cit. 1949

70 Idem

71 SHANNON, op. cit., 1948

72 WIENER, Norbert. Cybernetics: Control and communication in the animal and the machine. New York: MIT Press, Cambridge, MA e Wiley, 1948.

73 SHANNON, op. cit., 1948, op. cit., p. 34. [Tradução nossa].

74 SHANNON, op. cit., 1948, op. cit., p. 52. [Tradução nossa].

75 WIENER, op. cit., 1948.

76 WIENER, op. cit., 1956.

77 WIENER, op. cit., 1956, p. 48. [Tradução nossa].

78 ASH, Robert B. Information Theory. New York: Dover Publications, 1990, Prefácio, p. vi, tradução nossa. (Originalmente publicada por New York: Interscience Publishers, 1965).

79 PIERCE, John R. An introduction to information theory: symbols, signals and noise. New York: Dover Publications, 1961, p. 42. [Tradução nossa].

80 SHANNON; WEAVER, op. cit., 1949

81 SHANNON, Claude E.; WEAVER, Warren W. La théorie mathématique de la communication. Cassini, 2018, p. 125.

82 LUNDHEIM, Lars. On Shannon and "Shannon's Formula". Telektronikk, v. 98, n. 1, p. 20-29, 2002.

83 RIOUL, Olivier; MAGOSSI, José Carlos. On Shannon's formula and Hartley's rule: Beyond the mathematical coincidence. Entropy, v. 16, n. 9, p. 4.892-4.910, 2014.

84 RIOUL, Olivier; FLANDRIN, Patrick. Le dessein de Laplume. Proc. XXVlème Colloque GRETSI 2017, Juan-Les-Pins, France, 2017.

85 NEBEKER, op. cit., 1998.

86 FISHER, Ronald Aylmer. Theory of statistical estimation. In: Mathematical Proceedings of the Cambridge Philosophical Society. Cambridge University Press, 1925. v. 22, p. 700-725. Contributions to Mathematical Statistics, John Wiley \& Sons, New York, 1950, p. 11.

87 KULLBACK, Solomon. Information Theory and Statistics. John Wiley Sons, 1959, New York. Dover Publications, New York, 1997, p. 1-2.

88 Idem. PIERCE, op. cit., 1961.

89 SHANNON; WEAVER, op. cit., 1949.

90 SHANNON; WEAVER, op. cit., 2018.

91 SHANNON; WEAVER, op. cit., 1949.

[Artigo recebido em Dezembro de 2018. Aceito para publicação em Junho de 2019] 